\title{
Hierarchical living assembly: fabrication and visualization of multiblock microstructures
}

Xiujuan Shi, ${ }^{1,2,4, \#, *}$, Jun Zhang,,${ }^{1,5, \#}$ Junkai Liu, ${ }^{1}$ Xueqian Zhao, ${ }^{1}$ Haoran Wang, ${ }^{1}$ Peifa Wei, ${ }^{1,6}$ Xinlong Ni, ${ }^{7}$ Herman H.-Y. Sung, ${ }^{1}$ Ian D. Williams, ${ }^{1}$ Wai Kit Ng, ${ }^{8}$ Kam Sing Wong, ${ }^{8}$ Jacky W. Y. Lam, ${ }^{1}$ Lin Wang, ${ }^{2, *}$ Ben Zhong Tang ${ }^{1,3, *}$

${ }^{1}$ Department of Chemistry, Hong Kong Branch of Chinese National Engineering Research Center for Tissue Restoration and Reconstruction, and Guangdong-Hong Kong-Macao Joint Laboratory of Optoelectronic and Magnetic Functional Materials, The Hong Kong University of Science and Technology, Clear Water Bay, Kowloon, Hong Kong 999077, China. E-mail: tangbenz@ust.hk.

${ }^{2}$ Department of Clinical Laboratory, Research Center for Tissue Engineering and Regenerative Medicine, Union Hospital, Tongji Medical College, Huazhong University of Science and Technology, Wuhan 430022, China. Email: shixiujuan@hust.edu.cn; lin_wang@hust.edu.cn.

${ }^{3}$ Shenzhen Institute of Aggregate Science and Technology, School of Science and Engineering, The Chinese University of Hong Kong, Shenzhen, 2001 Longxiang Boulevard, Longgang District, Shenzhen City, Guangdong 518172, China. E-mail: tangbenz@cuhk.edu.cn.

${ }^{4}$ HKUST Shenzhen Research Institute, No. 9 Yuexing 1st RD, South Area Hi-tech Park, Nanshan, Shenzhen 518057, China.

${ }^{5}$ School of Materials and Chemical Engineering, Anhui Jianzhu University, Hefei 230601, China.

${ }^{6}$ Institutes of Physical Science and Information Technology, Key Laboratory of Structure and Functional Regulation of Hybrid Materials of Ministry of Education, Anhui University, Hefei, 230601, China.

${ }^{7}$ Key Laboratory of Macrocyclic and Supramolecular Chemistry of Guizhou Province, 
Guizhou University, Guizhou 550025, China.

${ }^{8}$ Department of Physics, The Hong Kong University of Science and Technology, Clear Water Bay, Kowloon, Hong Kong 999077, China.

${ }^{\#} \mathrm{X}$. Shi and J. Zhang contributed equally to this work.

\section{Abstract:}

Nature possesses a powerful ability to assemble multiple complex structures to fabricate hierarchical biological structures in a living-assembled way. However, it is still a huge challenge for artificial systems to fabricate and characterize hierarchical living assemblies with well-defined and controllable but complex structures. In this work, we proposed a new concept for the fabrication of multiblock fluorescent microcolumns, which relies on the cooperation between the controllable host-guest complexation based on cucurbit[8]uril (CB[8]) and the living assembly of nanotubular supramolecular polymers composed of $\mathrm{CB}[8]$ and $\mathrm{NaBr}$ in aqueous solution. By using the complexation of $\mathrm{CB}[8]$ with different guest numbers of luminogens with aggregation-induced emission (AIEgens) characteristics, and the difference in affinity between $\mathrm{CB}[8]$ and different types of AIEgens, the concentration-controlled and selfsorting-controlled sequential living assembly are realized, respectively. Correspondingly, multiblock fluorescent microcolumns with different fluorescence emission are fabricated, and the molecular structure of each fluorescent block is analyzed by single crystal X-ray diffraction measurement. In addition, the living assembly of multiblock fluorescent microcolumns is visualized, understood, and regulated with the aid of AIEgens. The method developed here is expected to be 
extended to more guest molecules of $\mathrm{CB}[8]$ and also provides a referential crystallization method for $\mathrm{CB}[8]$-based complexes.

\section{Introduction}

In biological systems, thousands of molecular building blocks assemble into hierarchical structures, such as actin filaments, microtubules, amyloid fibrils, muscle fibers, etc., through living assembly to carry out sophisticated biological processes. ${ }^{1}$ In muscle fiber cells, actin and myosin are actively assembled to form nanoscale multiband - alternating thin and thick filaments, which reassemble to form micronscale filamentous myofibrils. Then, bundles of muscle fibers are arranged to form centimeter-scale skeletal muscle tissue. ${ }^{2}$ Taking inspirations from nature, it is a very attractive scientific proposition to understand and develop biomimetic artificial hierarchical living assembly.

Indeed, great efforts have been made in the field of living assembly with the ability of epitaxial growth. It is widely known that living polymerization can retain chain-end fidelity, and it thus plays a vital role in the preparation of block copolymers. ${ }^{3-9}$ However, the construction of highly complex ordered structures remains a challenge for covalent polymerization, but can be achieved by supramolecular polymer chemistry. ${ }^{10}$ In recent years, living supramolecular assembly, such as living supramolecular polymerizations relying on $\pi$-stacking molecular amphiphiles, hydrogen bonding, metallophilic interactions, large dipole moment or $\beta$-sheet formation, ${ }^{11-26}$ and living crystallizationdriven self-assembly of block copolymers, ${ }^{27-31}$ have been used to fabricate a variety of 
ordered hierarchical assembly structures with remarkable complexity, like block fibers, block nanotubes, multiblock nanowires, 2D platelet co-micelles, etc. However, the supramolecular building units have complex structures that may not be well-defined or contain expensive noble metals, and most assemblies are only nanometer to submicron in width. Besides, the variety of living supramolecular assembly is still limited and much work remains to be done. The main reason is that the bottom-up controllable fabrication of living assembly materials with well-defined but complex structures presents a great challenge not only in fabrication but also in structural characterization.

In the past decades, supramolecular polymers have been extensively studied to facilitate its mechanistic interpretation at the molecular level. ${ }^{1,20,21,32-48}$ However, many well-established characterization methods for conventional polymers, such as gel permeation chromatography, mass spectrometry and so on, do not work well because the dilution effect and the detrimental interactions with matrixes may affect the structures of supramolecular polymers due to their dynamic nature. ${ }^{45,49}$ Commonly used spectroscopic techniques such as nuclear magnetic resonance, infrared, and Raman spectroscopy are limited in that they provide an ensemble average information on the molecular interactions, and cannot distinguish subtle variations at the microscopic scale. ${ }^{50}$ Scanning electron microscope, transmission electron microscope and atomic force microscope are also frequently applied, but the first two methods are not suitable for real-time monitoring of the assembly process, or even encounter sample change or damage during sample preparation, while the last method requires a long acquisition time. $^{49,51}$ In addition, it is not easy to grow micron-scale single crystals of 
supramolecular polymers that can be used for single crystal X-ray diffraction measurements. ${ }^{52}$ The difficulties of characterizing supramolecular polymer assembly make it difficult for people to understand the assembly mechanism and to achieve indepth and comprehensive control on the final assembly structures.

Is there any economical and practical characterization method that can visualize the supramolecular assembly process in situ and in real time, and provide real images and reliable results without additional sample preparation process? Fluorescence-based technologies can meet all the above requirements.,.$^{22,53-58}$ In the study of living supramolecular assembly, traditional fluorescent materials show inherent dilemma: their fluorescence is very bright in solution but is partially or completely quenched upon assembly and aggregation. Such a phenomenon is called aggregation-caused quenching (ACQ). ${ }^{59-61}$ On the contrary, the more materials with the characteristics of aggregationinduced emission (AIE) are assembled, the higher is their emission. Thus, AIE materials can reduce the fluorescence signal in solution but enhance it after assembly, ${ }^{62-74}$ making it an inherently promising tool for studying living supramolecular assembly. Our previous work found that AIEgen is not only a versatile probe for visualizing supramolecular assembly, but also an excellent guest for $\mathrm{CB}[8] \cdot{ }^{75}$ However, we acquired no large ordered assemblies so that we spent great efforts to infer the molecular structures of the supramolecular polymers through various solution characterization methods. We assume that the supramolecular polymer assembly based only on the hostguest interaction of $\mathrm{CB}[8]$ and AIEgen is so weak that prevents the further development of assembly. Thus, we hypothesize that large hierarchical structures are expected to 
form if multiple supramolecular interactions are used for assembly.

In this work, we aim to develop large and stable supramolecular assembly. According to the literature reports, we noted that $\mathrm{CB}[6]$ can form rod-shaped bulk crystals in $\mathrm{NaCl}$ solution through various supramolecular interactions. ${ }^{76}$ However, the cavity of $\mathrm{CB}[6]$ is too small to accommodate six-membered aromatic ring. ${ }^{77}$ Due to the large cavity size of $\mathrm{CB}[8]$, it can accommodate one or two six-membered aromatic rings and shows high binding affinity. ${ }^{78-81}$ We believe that if the host-guest interaction of $\mathrm{CB}[8]$ and AIEgen is further combined with the supramolecular interactions of $\mathrm{CB}[8]$ and salt, ${ }^{82,83}$ stable hierarchical supramolecular assembly from the molecular level to the nanometer level and then to the micron level may be obtained. Meanwhile, the fluorescence of AIEgens allows us to visualize the assembly process to gain reliable and useful assembly information. In this study, with the aid of AIEgens, we visualized the hierarchical assembly, understood the assembly mechanism, and realized the regulation of supramolecular assembly. This enables us to develop hierarchical living assembly for the fabrication of nano/micro multiblock microstructures in aqueous solution.

\section{Results and discussion}

\subsection{Microcolumns assembled from $\mathrm{CB}[8]$ and $\mathrm{NaBr}$ in the absence or presence of}

\section{AIEgens}

Since $\mathrm{CB}[8]$ shows a symmetrical structure with electrostatically negative carbonyl-fringed portal and electrostatically positive periphery (Fig. 1a), it is possible 
to form mesoscopic or even macroscopic crystals if the AIEgen-accommodated CB[8] complexes are connected in both the transverse and longitudinal direction through hierarchical supramolecular interactions. We initially checked the crystal assembly of $\mathrm{CB}[8]$ and $\mathrm{NaCl}$ in aqueous solution by using AIEgen, called CSPP, as a tracer. However, no large assembly was formed in solution (Supporting Fig. 1). It is possibly because the radius of $\mathrm{Cl}^{-}$ion is too small to match the lateral curvature of $\mathrm{CB}[8]$. Therefore, we further tried $\mathrm{NaBr}$ and $\mathrm{NaI}$, and found that assembly of $\mathrm{CB}[8]$ and salt is easier to form as the radius of halogen anion is much larger (Supporting Fig. 1). However, the assembly of $\mathrm{NaI}$ and $\mathrm{CB}[8]$ grows too fast, which is not conducive to the regulation and real-time monitoring of assembly. Therefore, $\mathrm{NaBr}$ is a better choice among $\mathrm{NaCl}$ and $\mathrm{NaI}$.
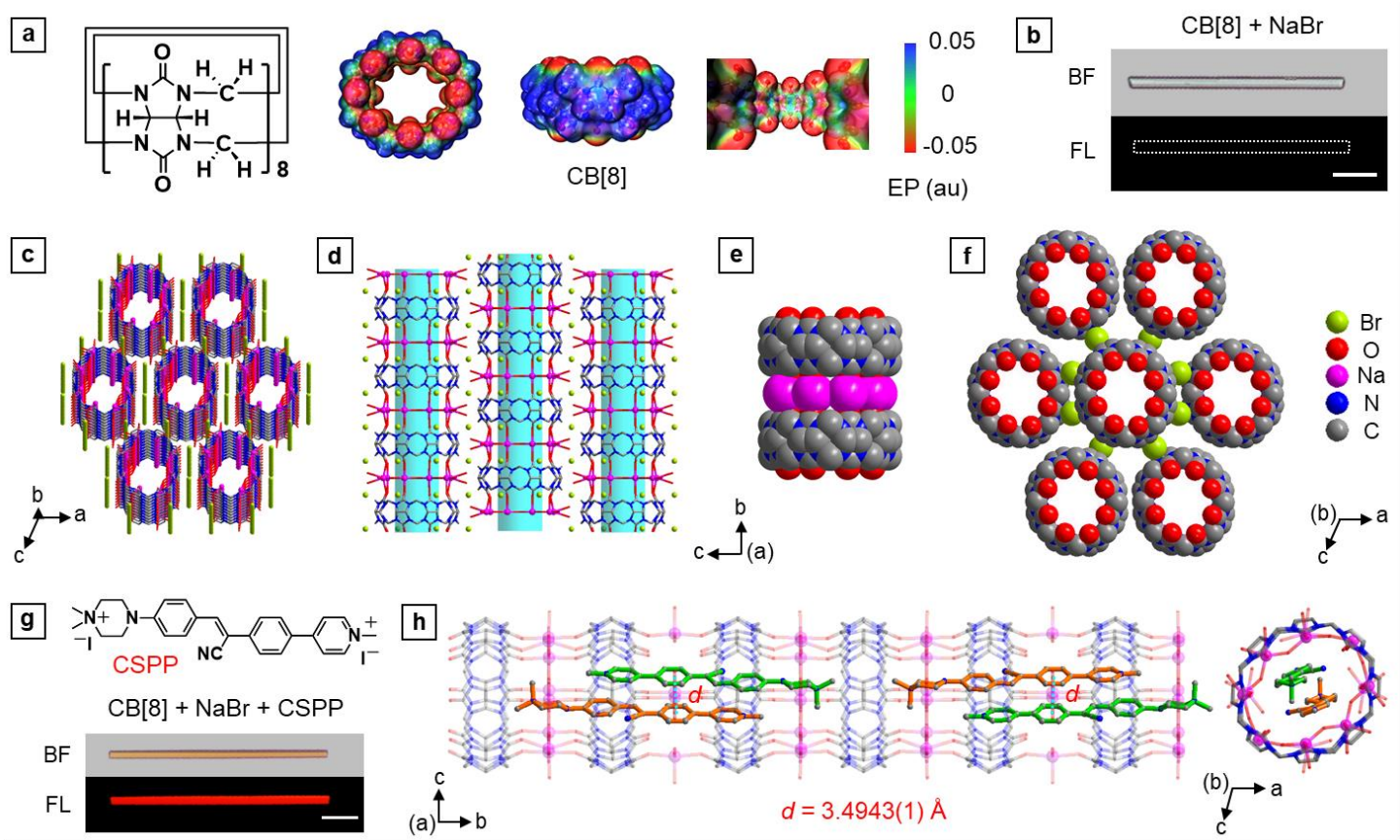

Figure 1 | Microcolumns assembled from $\mathrm{CB}[8]$ and $\mathrm{NaBr}$ in the absence or presence of AIEgens. a, Chemical structure and electrostatic potential (EP) maps of $\mathrm{CB}[8]$. b, Bright field (BF) and fluorescence (FL) images of a microcolumn assembled from $\mathrm{CB}[8]$ and $\mathrm{NaBr}$. The microcolumn is non-emissive and is thus framed with dotted 
line for clarity. $\lambda_{\text {ex: }}$ 400-440 nm; scale bar: $20 \mu \mathrm{m}$. c,d, Top (c) and side (d) views of the X-ray single crystal structure of the microcolumn. Nanocavities in the microcolumns are depicted in greenish blue bars. e,f, Simplified illustrations of the supramolecular interactions (e) between $\mathrm{Na}^{+}$cations and the portal of $\mathrm{CB}[8]$ in epitaxial direction, and (f) between $\mathrm{Br}^{-}$anions and the periphery of $\mathrm{CB}[8]$ in lateral direction. $\mathbf{g}$, Chemical structure of CSPP (an AIEgen) and BF and FL images of the microcolumn assembled from CSPP, CB[8] and NaBr. $\lambda$ ex: 400-440 nm; scale bar: $20 \mu \mathrm{m}$. h, X-ray single crystal structure of the red-emissive microcolumn. CSPP dimers are incorporated into the $\mathrm{CB}[8]-\mathrm{Na}^{+}$cation nanotubular polymer via supramolecular host-guest complexation.

$\mathrm{NaBr}$ is assembled with $\mathrm{CB}[8]$ to form colorless and non-emissive column-like crystals as shown in Fig. 1b. The X-ray single crystal analysis shows that the nanochannels are axially distributed in the non-occupied column composed of $\mathrm{NaBr}$ and $\mathrm{CB}[8]$ (Fig. 1c and 1d). In axial direction, the symmetric coordination interactions of $\mathrm{Na}^{+}$cation and the carbonyl oxygen of $\mathrm{CB}[8]$ portal axially connect the supramolecular assembly to form nanotube through $\left[\mathrm{O}_{\text {carbonyl }}-\mathrm{Na}-\mathrm{O}_{\text {carbonyl }}\right]$ coordination interactions at distances of 2.337(8), 2.367(8), 2.368(8) and 2.406(7) $\AA$ (Fig. 1e). Eight $\mathrm{Na}^{+}$cations are located above the eight carbonyl oxygens of $\mathrm{CB}[8]$ portal to form a circle. The $\mathrm{Na}^{+}$cations are also linked by structural water through $[\mathrm{Na}-$ Owater] coordination interactions to enhance the stability of the nanotube at a distance range of 2.00(3)-2.57(4) $\AA$. Since the nanotubes formed by $\mathrm{CB}[8]$ and $\mathrm{Na}^{+}$cations are straight and rigid, they are suitable for further lateral association with each other. ${ }^{84}$ Laterally, the $\mathrm{Br}^{-}$anions interact with the periphery of $\mathrm{CB}[8]$ through the $[\mathrm{Br} \cdots \mathrm{C}=\mathrm{O}]$ ion-dipole interactions with the electrostatically positive carbonyl carbon atoms of 
$\mathrm{CB}[8]$, and also through the special $[\mathrm{Br} \cdots \mathrm{H}-\mathrm{C}]$ hydrogen bonding with the methine and methylene groups of $\mathrm{CB}[8]$. The distances of these interactions fall in the range of 3.37(1)-3.697(8) $\AA$ and 3.11-3.45 $\AA$, respectively. At the same time, the $\mathrm{Br}^{-}$anions also interact with structural waters through $[\mathrm{Br} \cdots \mathrm{H}-\mathrm{O}]$ hydrogen bonds at a distance range of $2.38-2.95 \AA$. Thus, these ion-dipole interactions and hydrogen-bonding interactions connect one $\mathrm{CB}[8]$ in the center with surrounding six $\mathrm{CB}[8]$ s to serve as a repeating unit of the assembly, which connect the nanotubes together to form multistranded tubules (Fig. 1f). Detailed crystal information can be found in Supporting Fig.

\section{2 and Supporting Table 2.}

CSPP molecule has a rotatable donor-acceptor structure, and shows a remarkable effect of twisted intramolecular charge transfer. As the polarity of the surrounding environment decreases, its emission is red-shifted from $524 \mathrm{~nm}$ to $644 \mathrm{~nm}$ (Supporting

Fig. 3). Therefore, CSPP is expected to be used for sensitive tracking and functionalizing supramolecular assembly. After CSPP was mixed with $\mathrm{CB}[8]$ and $\mathrm{NaBr}$, red-emissive brownish-colored fluorescent microcolumns were observed by fluorescence imaging (Fig. 1g). Analysis of its single crystal structure by X-ray diffraction measurement reveals that CSPP dimers are distributed in the nanotube formed by $\mathrm{CB}[8]$ and $\mathrm{Na}^{+}$cations (Fig. 1h). The dimers have two conformations that can be transformed to each other by flipping horizontally. The distance between the centers of two parallel benzene rings in the dimer is 3.4943(1) $\AA$, indicating the existence of a strong $\pi-\pi$ interaction. The enhancement of conjugation between CSPP molecules in the dimer promotes fluorescence redshift. In addition, the guest molecule 
in the nanotube strengthens the axial connection of $\mathrm{CB}[8] \mathrm{s}$ through the ion-dipole interaction of its positively charged nitrogen and the carbonyl oxygen of $\mathrm{CB}[8]$, thereby improving the stability of CSPP dimer@CB[8]-NaBr crystals. This explains why CSPP dimer@ $\mathrm{CB}[8]-\mathrm{NaBr}$ crystals can be formed at lower concentrations than $\mathrm{CB}[8]-\mathrm{NaBr}$ crystals. In nanotubes, there are $8 \mathrm{Na}^{+}$cations located above each $\mathrm{CB}[8]$ portal in most cases, while there are only $4 \mathrm{Na}^{+}$cations located above $\mathrm{CB}[8]$ portal at the position of two parallel benzene rings (Fig. 1h). Perhaps it is because the two parallel benzene rings occupy too much space, there is not enough space to accommodate more $\mathrm{Na}^{+}$ cations. And the carbonyl oxygens of $\mathrm{CB}[8]$ portal that are not combined with $\mathrm{Na}^{+}$ cations form hydrogen bonds with structural water to enhance the stability of the nanotubes (Supporting Fig. 4). Like $\mathrm{CB}[8]-\mathrm{NaBr}$ crystal, the adjacent nanotubes in CSPP dimer@CB[8]-NaBr crystal are connected by $[\mathrm{Br} \cdots \mathrm{C}=\mathrm{O}]$ ion-dipole interactions and $[\mathrm{Br} \cdots \mathrm{H}-\mathrm{C}]$ hydrogen bonds of $\mathrm{Br}^{-}$anions with the periphery of $\mathrm{CB}[8]$, and the $[\mathrm{Br} \cdots \mathrm{H}-\mathrm{O}]$ hydrogen bonds of $\mathrm{Br}^{-}$anions with structural water (Supporting Fig. 4 and Supporting Table 3). Therefore, the axial and lateral connections inside the column and the encapsulating capability of $\mathrm{CB}[8]$ cavities indicate the nature of hierarchical assembly.

\subsection{Fabrication of triblock fluorescent microcolumns}

Since both the non-emissive $(\mathrm{N})$ column and the red-emissive $(\mathrm{R})$ column contain the framework composed of $\mathrm{CB}[8]$ and $\mathrm{NaBr}$, we assumed that $\mathrm{N}$ and $\mathrm{R}$ can form a block structure under certain conditions. If $\mathrm{CB}[8]$ is in excess, it may continue to grow at both ends of the already formed $\mathrm{R}$ to form a $\mathrm{N}-\mathrm{R}-\mathrm{N}$ triblock column. After 
optimizing the fabrication conditions, colorless-brownish-colorless triblock columns form and are observed in the bright field images (Fig. 2a and $\mathbf{2 b}$ ), indicating that the system is living assembly. Interestingly, by controlling the relative concentration of $\mathrm{CB}[8]$ to AIEgens and the evaporation rate, two types of triblock fluorescent columns, namely the $\mathrm{N}$ column at the end of $\mathrm{R}(\mathrm{N}-\mathrm{R}-\mathrm{N})$ and the yellow-emissive column at the end of R (Y-R-Y) are found (Fig. 2a and $\mathbf{2 b}$ ). Although they show similar bright field images, their fluorescence images are completely different. Compared with the preparation conditions of $\mathrm{N}-\mathrm{R}-\mathrm{N}$ triblock, $\mathrm{Y}-\mathrm{R}-\mathrm{Y}$ triblock fluorescent column is produced under the condition of reduced concentration of $\mathrm{CB}[8]$ and fast solution evaporation.

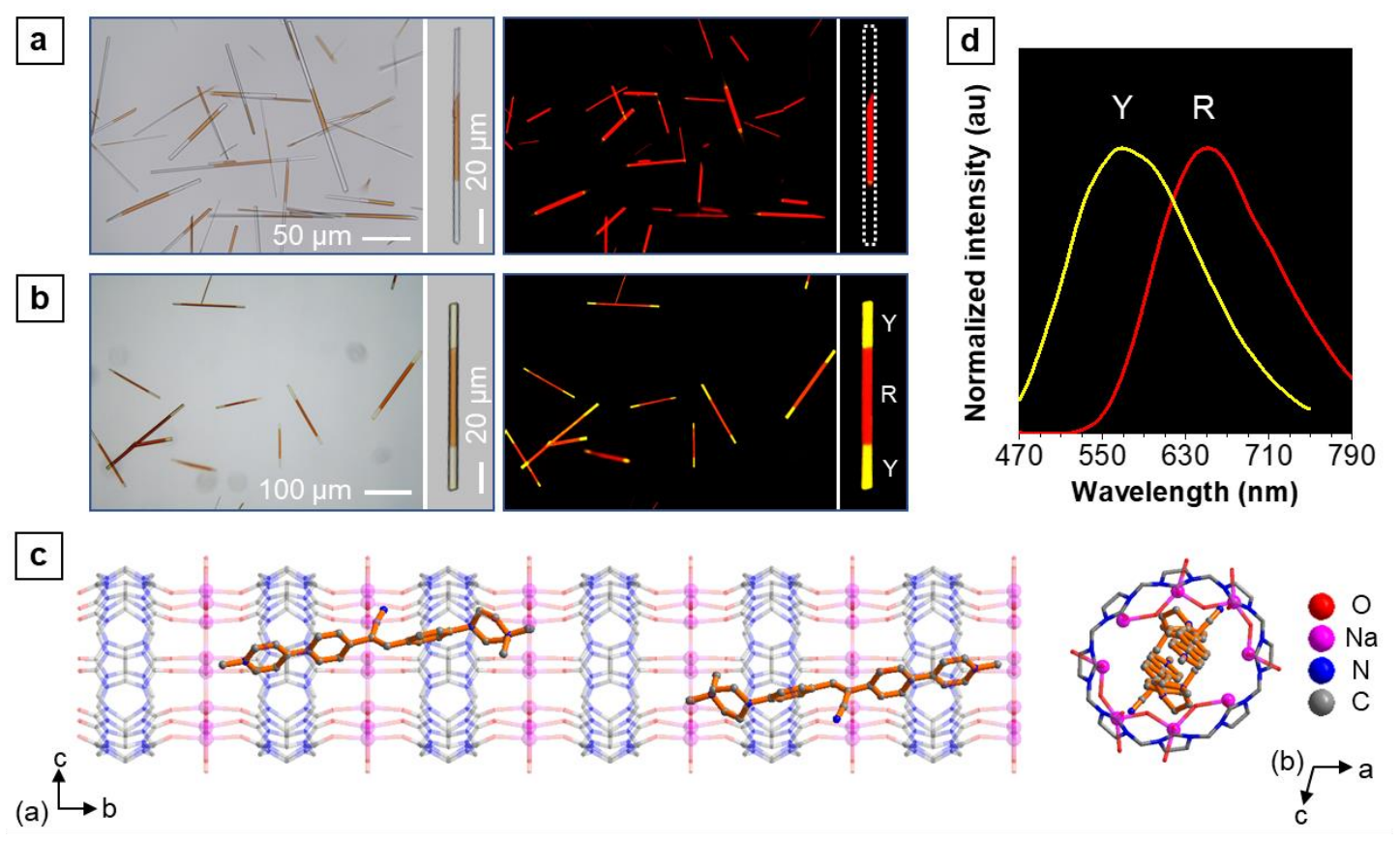

Figure 2 | Fabrication of 3-block fluorescent microcolumns through optimization of conditions. a,b, BF and $\mathrm{FL}$ images of (a) $\mathrm{N}-\mathrm{R}-\mathrm{N}$ and (b) $\mathrm{Y}-\mathrm{R}-\mathrm{Y}$ triblock microcolumns made from $\mathrm{CB}[8], \mathrm{NaBr}$ and $\mathrm{CSPP} . \mathrm{R}=$ red-emissive block, $\mathrm{Y}=$ yellowemissive block, $\mathrm{N}=$ non-emissive block. The fluorescent microcolumn in the right panel of $\mathbf{a}$ is framed with white dotted line for clarity; $\lambda_{\mathrm{ex}}: 400-440 \mathrm{~nm}$. c, X-ray single crystal structure of the Y block. CSPP monomers are incorporated into the $\mathrm{CB}[8]-\mathrm{Na}^{+}$ 
cation nanotubular polymer via host-guest complexation. d, FL spectra of the R and Y blocks shown in the right panel of $\mathbf{b}$ measured by a home-built optical system; $\lambda_{\text {ex }} 415$ nm.

It is well-known that the nanoconfined space of artificial macrocycles can make the encapsulated guest molecules to adopt a specific conformation, intermolecular distance or packing style. ${ }^{42,81,85-88}$ This may be the cause of the change in the emission behavior of AIEgens in $\mathrm{CB}[8]$ macrocycles. To figure out the molecular structure of the Y block, we obtained and studied its single crystal structure. X-ray diffraction analysis reveals that the crystal also shows a framework composed of $\mathrm{CB}[8]$ and $\mathrm{NaBr}$ (Supporting Fig. 5). The CSPP molecules are distributed in $\mathrm{CB}[8]-\mathrm{Na}^{+}$cation nanotubes in the form of monomer. There are four conformations of CSPP monomer, which can be converted to each other by vertical +/- horizontal flipping. Two of them are shown in Fig. 2c. and the rest are shown in Supporting Fig. 6. In the $\mathrm{Y}-\mathrm{R}-\mathrm{Y}$ triblock fluorescent column as shown in Fig. 2b, the fluorescence emission maximum of $\mathrm{Y}$ is located at $570 \mathrm{~nm}$, while that of $\mathrm{R}$ is $650 \mathrm{~nm}$ (Fig. 2d). The different emission of $\mathrm{Y}$ and $\mathrm{R}$ can be explained by using theoretical calculation on the frontier molecular orbitals and the potential energy surface as shown in Supporting Fig. 7 and Supporting Fig. 8. It is revealed that the energy gap between the HOMO and LUMO of CSPP monomer@CB[8] is higher than that of CSPP dimer@CB[8] (Supporting Fig. 7). This is mainly because the torsion angle of CSPP monomer in the hydrophobic CB[8] cavity is close to right angle $\left(-96.59^{\circ}\right)$, which results in a high potential energy surface and blue-shifted emission. However, the torsion angle of CSPP dimer in CB[8] cavity 
is very small $\left(-1.04^{\circ}\right)$, which leads to a small potential energy surface (Supporting Fig. 8). Together with its enhanced $\pi-\pi$ conjugation, CSPP dimer@CB[8] shows a redshifted emission.

In addition, the formation mechanism of $\mathrm{Y}-\mathrm{R}-\mathrm{Y}$ and $\mathrm{N}-\mathrm{R}-\mathrm{N}$ triblock fluorescent columns was analyzed. The sequential living assembly process of $\mathrm{Y}-\mathrm{R}-\mathrm{Y}$ triblock was monitored and demonstrated by fluorescence imaging (Supporting Fig. 9). In solution, the $\mathrm{CB}[8]$ cavity of CSPP monomer@CB[8] contains more high-energy water, resulting in its structural instability, while CSPP dimer@CB[8] is relatively more stable and preferentially exists in solution. ${ }^{89}$ Thus, we can concluded that when the CSPP concentration is relatively high, the CSPP dimer@CB[8]-NaBr polymers are predominant in solution. On the other hand, when $\mathrm{CB}[8]$ is in excess, the CSPP monomer@CB[8]-NaBr and $\mathrm{CB}[8]-\mathrm{NaBr}$ polymers preferentially exist in solution after the consumption of most CSPP to produce R. And after the solution evaporation to reach the saturation of $\mathrm{CB}[8]$, both can be assembled and fixed. By using PL and UV-vis spectroscopy to track the change of the solution that is similar to the residual solution after the formation of $\mathrm{R}$ upon gradual addition of $\mathrm{CB}[8]$, the assembly process of CSPP monomer@ $\mathrm{CB}[8]-\mathrm{NaBr}$ and $\mathrm{CB}[8]-\mathrm{NaBr}$ polymers was demonstrated (Supporting Fig. 11). It is worth noting that the CSPP dimer@CB[8]-NaBr polymer and the CSPP monomer@ $\mathrm{CB}[8]-\mathrm{NaBr}$ polymer can be interconverted to each other under certain conditions, and such transformations were directly visualized and tracked through real-time fluorescence imaging as shown in Supporting Fig. 13, but failed to be observed by bright field imaging and electron microscopic imaging. To fabricate $\mathrm{Y}_{-}$ 
$\mathrm{R}-\mathrm{Y}$ triblock, the solution was evaporated rapidly. In this case, CSPP monomer@CB[8]-NaBr polymers do not have sufficient time to transform into CSPP dimer@ $\mathrm{CB}[8]-\mathrm{NaBr}$ polymers. When $\mathrm{CB}[8]$ reaches saturation, they epitaxially grow at both ends of $\mathrm{R}$ to form $\mathrm{Y}-\mathrm{R}-\mathrm{Y}$ triblock. For the fabrication of $\mathrm{N}-\mathrm{R}-\mathrm{N}$ triblock, the solution was evaporated more slowly. Therefore, in the presence of more $\mathrm{CB}[8]$ and under the condition of slow solution evaporation, the CSPP monomer@CB[8]- $\mathrm{NaBr}$ polymers have enough time to transform into the thermodynamically more stable CSPP dimer@CB[8]-NaBr polymers, whose assembly makes the R column continue to grow, eventually leading to the complete consumption of CSPP. Subsequently, the excess $\mathrm{CB}[8]$ continues to be epitaxially assembled with $\mathrm{NaBr}$ at both ends of $\mathrm{R}$ to generate $\mathrm{N}-\mathrm{R}-\mathrm{N}$ triblock.

Therefore, the formation of $\mathrm{N}-\mathrm{R}-\mathrm{N}$ and $\mathrm{Y}-\mathrm{R}-\mathrm{Y}$ triblock is mainly affected by the relative concentration of $\mathrm{CB}[8]$ and the evaporation rate of solution. Considering that the one-pot assembly of fluorescent multiblock columns is a concentration-controlled sequential living assembly process, it is probably affected by the $\mathrm{CB}[8]$ concentration, the relative ratio of guest to $\mathrm{CB}[8]$, the guest concentration, the $\mathrm{NaBr}$ concentration, and the evaporation rate of solution. Adjustment of these factors is expected to produce more complex assemblies.

\subsection{One-pot multiblock living assembly}

Nature always uses multiple components to build hierarchical complex biological assemblies. By drawing inspiration from nature, we hope to use N, R, and Y columns as building blocks to create more complex structures through the one-pot living 
assembly as now we know the conditions under which they are formed. Taking the formation conditions of $\mathrm{N}-\mathrm{R}-\mathrm{N}$ triblock (3-block) into account, we slightly increased the relative ratio of AIEgen to $\mathrm{CB}[8]$ while keeping the amount of $\mathrm{CB}[8]$ in excess, and also speed up the evaporation process. When $\mathrm{CB}[8]$ reaches saturation after $\mathrm{R}$ is formed, the $\mathrm{CB}[8]-\mathrm{NaBr}$ polymers continue to grow at the R terminals to form $\mathrm{N}$. With the consumption of $\mathrm{CB}[8]$, the concentration of CSPP monomer@CB[8]-NaBr polymers increases relatively, and their continued growth at the end of $\mathrm{N}-\mathrm{R}-\mathrm{N}$ leads to form the penta-block (5-block) fluorescent microcolumn $\mathrm{Y}-\mathrm{N}-\mathrm{R}-\mathrm{N}-\mathrm{Y}$ (Fig. 3a). In addition, based on the formation conditions of $\mathrm{Y}-\mathrm{N}-\mathrm{R}-\mathrm{N}-\mathrm{Y}$ 5-block, we increased the concentration of $\mathrm{CB}[8]$. Therefore, after $\mathrm{Y}-\mathrm{N}-\mathrm{R}-\mathrm{N}-\mathrm{Y}$ 5-block is generated, there is still residual $\mathrm{CB}[8]$ in the system, which continues to grow $\mathrm{N}$ at the terminals of $\mathrm{Y}-\mathrm{N}-$ $\mathrm{R}-\mathrm{N}-\mathrm{Y}$ to lead to the formation of hepta-block (7-block) fluorescent microcolumn $\mathrm{N}-$ $\mathrm{Y}-\mathrm{N}-\mathrm{R}-\mathrm{N}-\mathrm{Y}-\mathrm{N}$ (Fig. 3b). 


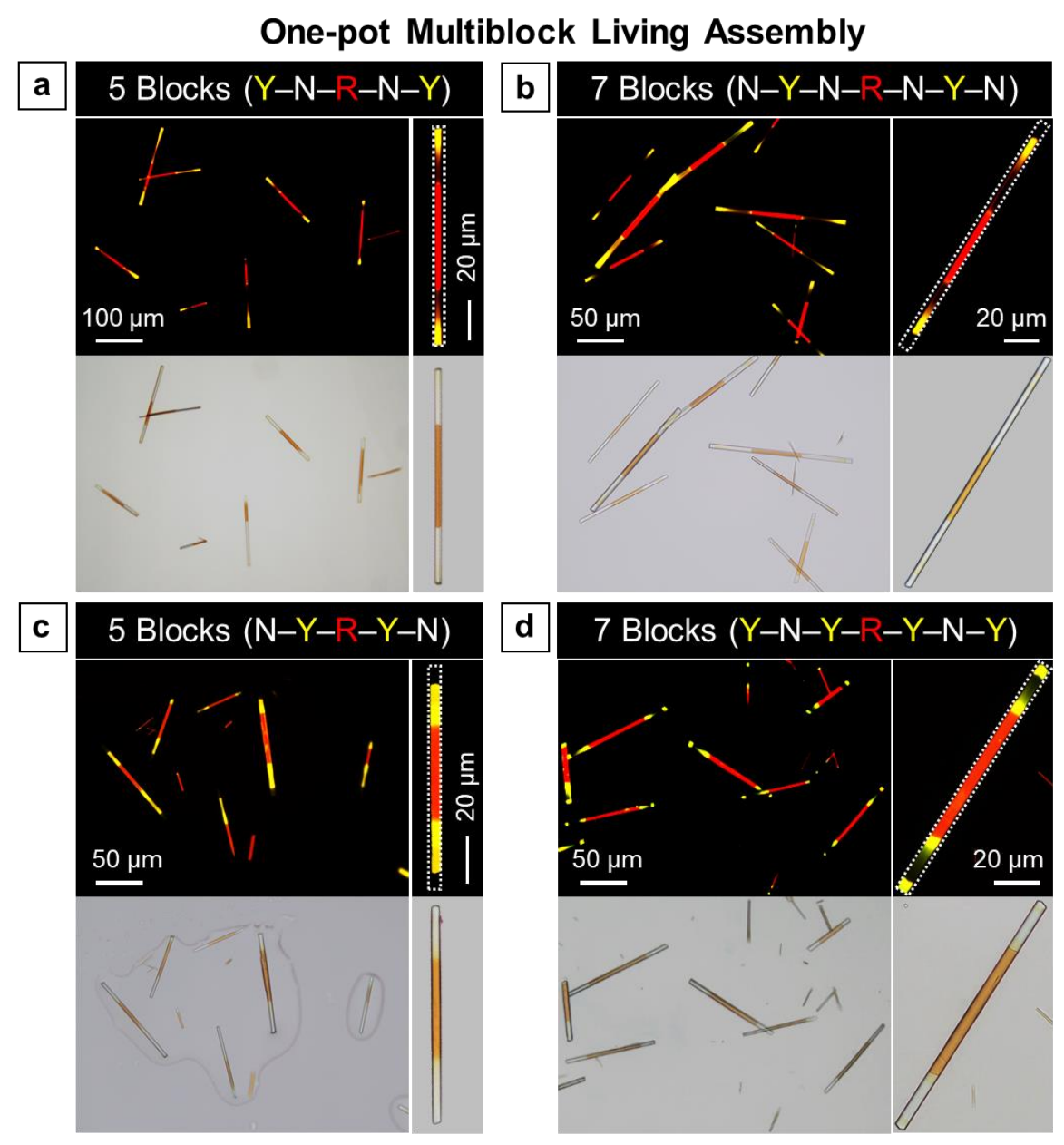

Figure 3 | Fabrication of 5-block and 7-block fluorescent microcolumns through one-pot living assembly. FL and BF images of the microcolumns with (a) $\mathrm{Y}-\mathrm{N}-\mathrm{R}-\mathrm{N}-$ $\mathrm{Y}$ and (c) N-Y-R-Y-N penta-block and (b) N-Y-N-R-N-Y-N and (d) $\mathrm{Y}-\mathrm{N}-\mathrm{Y}-\mathrm{R}-$ Y-N-Y hepta-block. $\lambda_{\text {ex: }} 400-440 \mathrm{~nm}$. The fluorescent microcolumns are framed with white dotted lines for clarity. $\mathrm{R}=$ red-emissive block, $\mathrm{Y}=$ yellow-emissive block, $\mathrm{N}=$ non-emissive block.

In addition, on the basis of the formation condition of $\mathrm{Y}-\mathrm{R}-\mathrm{Y}$ 3-block, we increased the relative ratio of $\mathrm{CB}[8]$ to AIEgen. After $\mathrm{Y}-\mathrm{R}-\mathrm{Y}$ is generated, the remaining $\mathrm{CB}[8] \mathrm{s}$ in the system continue to assemble at the terminals of $\mathrm{Y}-\mathrm{R}-\mathrm{Y}$ to generate $\mathrm{N}$, leading to the formation of $\mathrm{N}-\mathrm{Y}-\mathrm{R}-\mathrm{Y}-\mathrm{N}$ 5-block fluorescent microcolumns (Fig. 3c). Moreover, based on the formation conditions of N-Y-R-Y- 
$\mathrm{N}$ 5-block, we increased the concentration of AIEgens. After $\mathrm{N}-\mathrm{Y}-\mathrm{R}-\mathrm{Y}-\mathrm{N}$ is generated, there is still CSPP monomer@CB[8]-NaBr polymers in the system, which continues to grow at the ends of $\mathrm{N}-\mathrm{Y}-\mathrm{R}-\mathrm{Y}-\mathrm{N}$ to produce $\mathrm{Y}$, thereby forming $\mathrm{Y}-\mathrm{N}-\mathrm{Y}-\mathrm{R}-\mathrm{Y}-\mathrm{N}-\mathrm{Y}$ 7-block fluorescent microcolumns (Fig. 3d).

\subsection{Stepwise sequential multiblock living assembly}

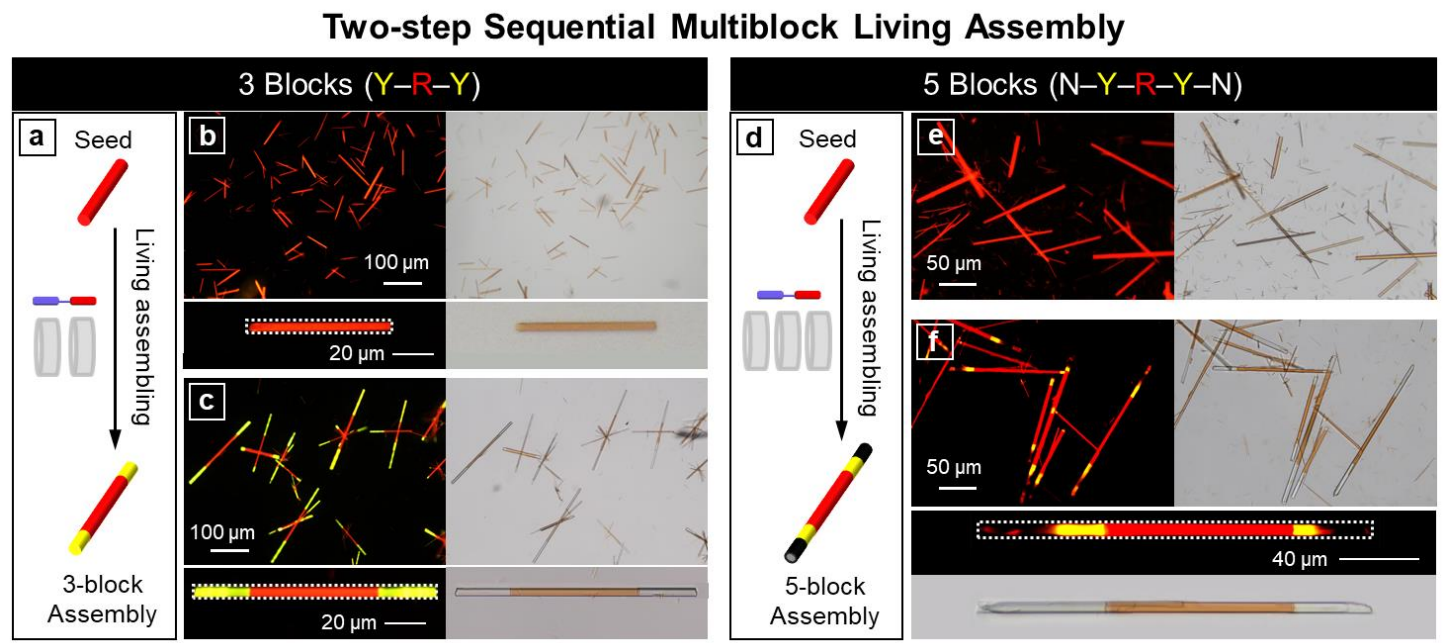

Figure 4 | Fabrication of multiblock fluorescent microcolumns through stepwise sequential living assembly. a, Schematic illustration of 2-step living assembly to fabricate 3-block fluorescent microcolumns. b,c, FL and BF images of (b) R seeds and (c) $\mathrm{Y}-\mathrm{R}-\mathrm{Y}$ 3-block microcolumns formed after addition of the growth solution and slow evaporation. d, Schematic illustration of 2-step living assembly to fabricate 5block fluorescent microcolumns. e,f, FL and BF images of (e) R seeds and (f) N-Y-RY-N 5-block microcolumns formed after addition of the growth solution and slow evaporation. $\lambda_{\text {ex: }}$ 400-440 $\mathrm{nm}$. The fluorescent microcolumns are framed with white dotted lines for clarity. $\mathrm{R}=$ red-emissive block, $\mathrm{Y}=$ yellow-emissive block, $\mathrm{N}=$ nonemissive block.

Stepwise assembly by adding dormant building blocks in sequence is a widely used method to prepare supramolecular block copolymers, multiblock fibers or 
multiblock fluorescent rods after a nucleation-elongation growth process. ${ }^{90-92}$ Now we have successfully fabricated 3-block, 5-block and 7-block fluorescent microcolumns through one-pot living assembly, we also want to fabricate multiblock fluorescent microcolumns through conventional stepwise living assembly as to prove the versatility of this assembly system.

In the first step, $\mathrm{R}$ seeds are prepared after evaporating the solution. Then, an appropriate amount of growth solution is added to R seeds, as illustrated in the schemes of Fig. 4a and 4d. The growth solution contains low concentration of CSPP, excessive $\mathrm{CB}[8]$ and a high concentration of $\mathrm{NaBr}$. After adding the growth solution, the system mainly contains the building units of CSPP monomer@CB[8]-NaBr polymers and $\mathrm{CB}[8]-\mathrm{NaBr}$ polymers for living assembly. The formed $\mathrm{R}$ seeds are shown in Fig. $\mathbf{4 b}$ and 4e. After adding the growth solution to the R seeds, the mixture was evaporated. In the presence of a slight excess of $\mathrm{CB}[8]$ and under the condition of rapid evaporation, the CSPP monomer@CB[8]-NaBr polymers epitaxially grow at the ends of $\mathrm{R}$ to form Y-R-Y 3-blocks (Fig. 4c). For the fabrication of N-Y-R-Y-N 5-block microcolumns, a growth solution was added to the R seeds, and the mixture was evaporated. Under the conditions of excessive $\mathrm{CB}[8]$ and rapid solution evaporation, $\mathrm{Y}$ columns are first formed at the ends of $\mathrm{R}$ until all the CSPP monomer@ $\mathrm{CB}[8]-\mathrm{NaBr}$ polymers are completely consumed. Then, the excessive $\mathrm{CB}[8]$ continues to assemble epitaxially at the ends of $\mathrm{Y}-\mathrm{R}-\mathrm{Y}$ to generate $\mathrm{N}-\mathrm{Y}-\mathrm{R}-\mathrm{Y}-\mathrm{N}$ 5-blocks (Fig. 4f).

2.5. One-pot sequential self-sorting multiblock living assembly from different types of AIEgen guests 
Since $\mathrm{CB}[8]$ has a variety of guest types, ${ }^{33,78,93} \mathrm{CB}[8]-\mathrm{NaBr}$ polymers may serve as a framework to accommodate other guests to form microcolumns with different fluorescence emission. Because of the difference in affinity between different guests and $\mathrm{CB}[8]$, it is possible to fabricate multiblock fluorescent microcolumns through supramolecular self-sorting-controlled sequential multiblock living assembly. To prove this concept, we chose another type of AIEgen, called CSPyP, whose structure is like CSPP but possesses pyridine instead of pyridinium group (Fig. 5a). As we expected, CSPyP can assemble with $\mathrm{CB}[8]$ and $\mathrm{NaBr}$ to form microcolumns (Fig. 5a) with green emission (G) at $558 \mathrm{~nm}$ (Supporting Fig. 14). Analysis of its single crystal structure by X-ray diffraction measurement (Supporting Fig. 15) found that CSPyP dimers are distributed in $\mathrm{CB}[8]-\mathrm{Na}^{+}$cation nanotubes probably in the form of $\mathrm{AA}, \mathrm{BB}$ and $\mathrm{AB}$ packings (Fig. 5b). In addition to ion-dipole interactions and hydrophobic interactions with $\mathrm{CB}[8]$, CSPyP also shows strong $\mathrm{C}-\mathrm{H} \cdots \pi$ interactions in the dimers of AA and AB packings (Supporting Fig. 16).

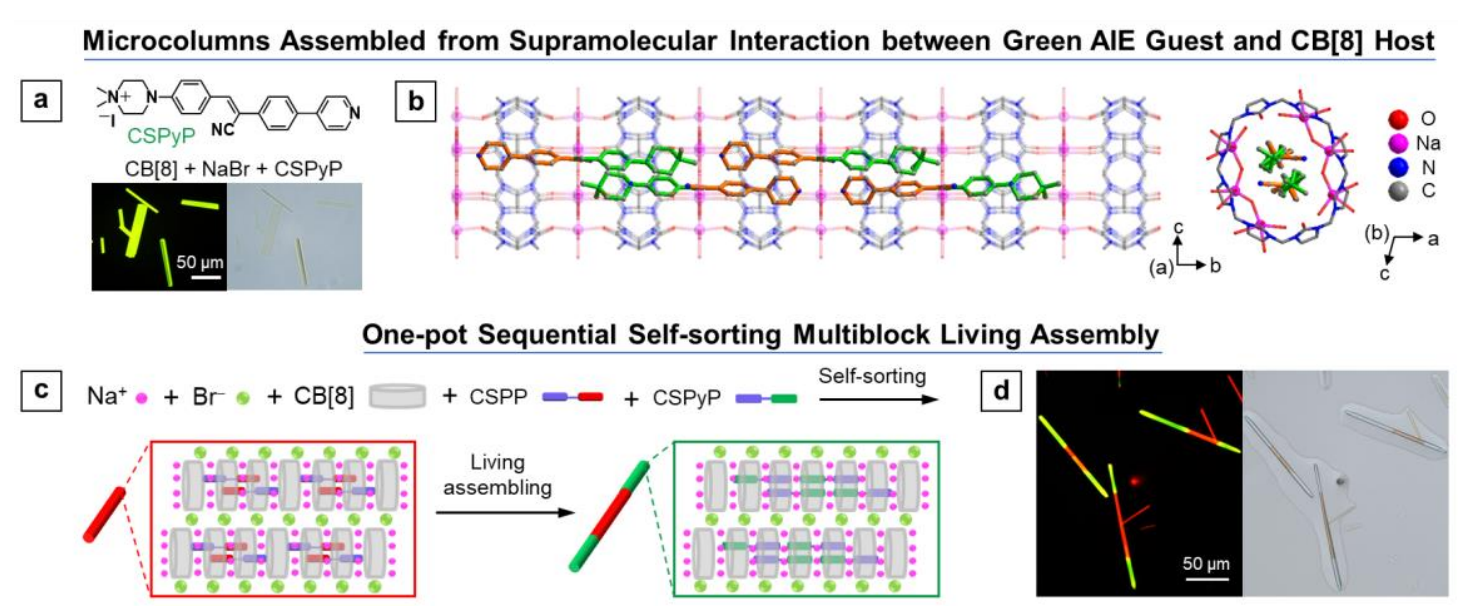

Figure 5 | One-pot sequential self-sorting multiblock living assembly from different types of AIEgen guests. a, Chemical structure of CSPyP and FL and BF images of green-emissive microcolumns assembled from $\mathrm{CB}[8], \mathrm{NaBr}$ and CSPyP; $\lambda_{\text {ex: }}$ : 400-440 nm. b, X-ray single crystal structure of the green-emissive microcolumn. c, 
Schematic illustration of one-pot fabrication of $\mathrm{G}-\mathrm{R}-\mathrm{G}$ 3-block fluorescent microcolumns from a mixture of CSPP, CSPyP, $\mathrm{CB}[8]$ and $\mathrm{NaBr} . \mathrm{G}=$ green-emissive block, $\mathrm{R}$ = red-emissive block. $\mathbf{d}$, FL and BF images of G-R-G 3-block microcolumns; $\lambda_{\text {ex }}: 400-440 \mathrm{~nm}$.

Since the portal of $\mathrm{CB}[\mathrm{n}] \mathrm{s}$ is highly electrostatically negative and the inner cavity of $\mathrm{CB}[\mathrm{n}] \mathrm{s}$ is remarkably hydrophobic, $\mathrm{CB}[\mathrm{n}] \mathrm{s}$ show a preference to dicationic species in which the charges are separated by hydrophobic regions. ${ }^{77}$ Because CSPP has two positive charges separated by hydrophobic aromatic groups, the host-guest affinity of CSPP with $\mathrm{CB}[8]$ is stronger than that of CSPyP, because it possesses less positive charge than CSPP. Therefore, it is assumed that CSPP dimer@CB[8]-NaBr polymers are first assembled to form R columns. After they are completely consumed, the CSPyP dimer@CB[8]-NaBr polymers will continue to grow epitaxially at the $\mathrm{R}$ terminals to generate 3-block columns as illustrated in Fig. 5c. To verify the hypothesis, we mixed CSPP and CSPyP at a ratio of 1:1 as the guest. The fluorescence and bright field images of the formed assemblies in Fig. 5d indicate that the mixed guests do indeed assemble with $\mathrm{CB}[8]$ and $\mathrm{NaBr}$ to form $\mathrm{G}-\mathrm{R}-\mathrm{G}$ 3-block fluorescent microcolumns. Therefore, it is proved that the mixture can undergo supramolecular self-sorting to form CSPP dimer@CB[8]-NaBr polymers and CSPyP dimer@CB[8]-NaBr polymers. The former is more stable and can be assembled first, and the latter is assembled at the end of $\mathrm{R}$ formed by the former (CSPP dimer@CB[8]-NaBr polymers), thus realizing the selfsorting-controlled sequential multiblock living assembly in one pot. 


\section{Conclusions}

In the present work, the nanotubular supramolecular polymer formed by the supramolecular interactions of $\mathrm{CB}[8]$ macrocycle and $\mathrm{NaBr}$ is used as the framework, which is axially connected by $\mathrm{CB}[8]$ and $\mathrm{Na}^{+}$cations and is further laterally connected by $\mathrm{Br}^{-}$anions to form a large columnar assembly. And its ability of continuous epitaxial growth realizes the living assembly. In addition, the hierarchical living assembly of the multiblock microcolumns composed by $\mathrm{CB}[8], \mathrm{NaBr}$ and AIEgens in aqueous solution is visualized by the fluorescence properties of AIEgens. By making use of the complexation between $\mathrm{CB}[8]$ and different numbers of AIEgen guests $(2,1,0)$, the concentration-controlled sequential living assembly is realized to achieve multiblock fluorescent microcolumns with different fluorescence emission, such as $\mathrm{Y}-\mathrm{R}-\mathrm{Y}$ and $\mathrm{N}-$ R-N 3-blocks, N-Y-R-Y-N and $\mathrm{Y}-\mathrm{N}-\mathrm{R}-\mathrm{N}-\mathrm{Y}$ 5-blocks, $\mathrm{Y}-\mathrm{N}-\mathrm{Y}-\mathrm{R}-\mathrm{Y}-\mathrm{N}-\mathrm{Y}$ and $\mathrm{N}-$ $\mathrm{Y}-\mathrm{N}-\mathrm{R}-\mathrm{N}-\mathrm{Y}-\mathrm{N}$ 7-blocks. In addition, by using the difference in affinity between CB[8] and different types of AIEgen guests, the self-sorting-controlled sequential living assembly is achieved, leading to the fabrication of $\mathrm{G}-\mathrm{R}-\mathrm{G}$ 3-block fluorescent microcolumns. Furthermore, the molecular structure of each fluorescent block is analyzed by single crystal X-ray diffraction measurement.

To our knowledge, the complex multiblock assemblies made by artificial macrocycle and ordinary salt in aqueous solution are unprecedented so far. This method is very simple, and the raw materials are economical and non-toxic. Since $\mathrm{CB}[8]$ can accommodate guests of many kinds, our method is expected to be extended to more guest molecules to construct more multiblock microcolumns with different fluorescent 
colors, which has a broad application prospect in anti-counterfeiting fluorescent barcode. In addition, due to the importance of single crystals in structural analysis, the preparation of large crystals of supramolecular host-guest complex has always been a difficult task. In this work, the crystallization of the complex of $\mathrm{CB}[8]$ and its guest is easily achieved by using the nanotubular supramolecular polymers formed by $\mathrm{CB}[8]$ macrocycle and $\mathrm{NaBr}$ as a framework. Therefore, our research also provides a referential crystallization method for $\mathrm{CB}[8]$-based complexes.

Accession Codes. CCDC 2034452, 2110796, 2034451 and 2110704 contain the supplementary crystallographic data of this paper. These data can be obtained free of charge via www.ccdc.cam.ac.uk/data_request/cif, or by emailing data_request@,ccdc.cam.ac.uk, or by contacting The Cambridge Crystallographic Data Centre, 12 Union Road, Cambridge CB2 1EZ, UK; fax: +44 1223336033.

\section{Acknowledgements}

This project was financially supported by the National Natural Science Foundation of China Grants (21788102, 82072167, and 81873931), the Innovation and Technology Commission (ITC-CNERC14SC01), the Research Grants Council of Hong Kong (16307020, C6014-20W, and N-HKUST609/19), the Science and Technology Plan of Shenzhen (JCYJ20170818113538482), and the Research and Development program from Hubei Province (2021ACB001). 


\section{Author Contributions}

X. Shi and J. Zhang contributed equally to this work. Dr. X. Shi did the experiments, analyzed the data, wrote the manuscript, and supported the funding. Prof. J. Zhang gave suggestions for crystal growth and analyzed the raw data of single crystal X-ray diffraction measurements. Dr. J. Liu did the computational calculations. Dr. X. Zhao, Dr. H. Wang and Prof. P. Wei made suggestions for writing the manuscript. Prof. X. Ni provided CB[8]. Dr. Herman H.-Y. Sung and Prof. Ian D. Williams did the single crystal X-ray diffraction measurements. Mr. W. K. Ng and Prof. K. S. Wong measured the fluorescence emission by using a home-built optical system. Dr. Jacky W. Y. Lam revised the manuscript. Prof. L. Wang supported the funding. Prof. B. Z. Tang supported the funding, revised the figures, and conceived the outline of the manuscript.

\section{Competing Interests statement}

The authors declare no competing interests.

\section{References}

1. Krieg, E., Bastings, M.M.C., Besenius, P. \& Rybtchinski, B. Supramolecular polymers in aqueous media. Chem. Rev. 116, 2414-2477 (2016).

2. Davies, K.E. \& Nowak, K.J. Molecular mechanisms of muscular dystrophies: Old and new players. Nat. Rev. Mol. Cell. Biol. 7, $762-773$ (2006).

3. Kamigaito, M., Maeda, Y., Sawamoto, M. \& Higashimura, T. Living cationic polymerization of isobutyl vinyl ether by hydrogen chloride/Lewis acid initiating systems in the presence of salts: In-situ direct NMR analysis of the growing species. Macromolecules 26, 1643 (1993).

4. M., S. 'Living' polymers. Nature 178, 1168-1169 (1956).

5. Nikolaev, A., Lu, Z., Chakraborty, A., Sepunaru, L. \& de Alaniz, J.R. Interconvertible living radical and cationic polymerization using a dual photoelectrochemical catalyst. J. Am. Chem. Soc. 143, 12278-12285 (2021). 
6. Moad, G., Rizzardo, E. \& Thang, S.H. Toward living radical polymerization. Acc. Chem. Res. 41, 1133-1142 (2008).

7. Braunecker, W.A. \& Matyjaszewski, K. Controlled/living radical polymerization: Features, developments, and perspectives. Prog. Polym. Sci. 32, 93-146 (2007).

8. Perrier, S. 50th anniversary perspective: RAFT polymerization - a user guide. Macromolecules 50, 7433-7447 (2017).

9. Grubbs, R.B. \& Grubbs, R.H. 50th anniversary perspective: Living polymerization-emphasizing the molecule in macromolecules. Macromolecules 50, 6979-6997 (2017).

10. Qin, B., et al. Supramolecular polymer chemistry: From structural control to functional assembly. Prog. Polym. Sci. 100, 101167 (2020).

11. Ogi, S., Sugiyasu, K., Manna, S., Samitsu, S. \& Takeuchi, M. Living supramolecular polymerization realized through a biomimetic approach. Nat. Chem. 6, 188-195 (2014).

12. Hu, K., et al. Fabrication of single-handed nanocoils with controlled length via a living supramolecular self-assembly. Chem. Mater. 31, 1403-1407 (2019).

13. Wagner, W., Wehner, M., Stepanenko, V. \& Würthner, F. Supramolecular block copolymers by seeded living polymerization of perylene bisimides. J. Am. Chem. Soc. 141, 12044-12054 (2019).

14. Wagner, W., Wehner, M., Stepanenko, V., Ogi, S. \& Würthner, F. Living supramolecular polymerization of a perylene bisimide dye into fluorescent Jaggregates. Angew. Chem. Int. Ed. Engl. 56, 16008-16012 (2017).

15. Shyshov, O., et al. Living supramolecular polymerization of fluorinated cyclohexanes. Nat. Commun. 12, 3134 (2021).

16. Zhang, W., et al. Supramolecular linear heterojunction composed of graphite-like semiconducting nanotubular segments. Science 334, 340-343 (2011).

17. Chen, Z., et al. Solvent-free autocatalytic supramolecular polymerization. Nat. Mater., https://doi.org/10.1038/s41563-41021-01122-z (2021).

18. Wehner, M. \& Würthner, F. Supramolecular polymerization through kinetic pathway control and living chain growth. Nat. Rev. Chem. 4, 38-53 (2020).

19. Kemper, B., et al. Kinetically controlled stepwise self-assembly of $\mathrm{Au}(\mathrm{I})$ metallopeptides in water. J. Am. Chem. Soc. 140, 534-537 (2018).

20. Korevaar, P.A., et al. Pathway complexity in supramolecular polymerization. Nature 481, 492-496 (2012).

21. Helmers, I., Ghosh, G., Albuquerque, R.Q. \& Fernández, G. Pathway and length control of supramolecular polymers in aqueous media via a hydrogen bonding lock. Angew. Chem. Int. Ed. Engl. 60, 4368-4376 (2021).

22. Aliprandi, A., Mauro, M. \& De Cola, L. Controlling and imaging biomimetic selfassembly. Nat. Chem. 8, 10-15 (2016).

23. Wan, Q., Xia, J., Lu, W., Yang, J. \& Che, C.M. Kinetically controlled selfassembly of phosphorescent $\mathrm{Au}(\mathrm{III})$ aggregates and ligand-to-metal-metal charge transfer excited state: A combined spectroscopic and DFT/TDDFT study. J. Am. Chem. Soc. 141, 11572-11582 (2019).

24. Wan, Q., To, W.P., Yang, C. \& Che, C.M. The metal-metal-to-ligand charge 
transfer excited state and supramolecular polymerization of luminescent pincer Pd(II)-isocyanide complexes. Angew. Chem. Int. Ed. Engl. 57, 3089-3093 (2018).

25. Pal, A., et al. Controlling the structure and length of self-synthesizing supramolecular polymers through nucleated growth and disassembly. Angew. Chem. Int. Ed. Engl. 54, 7852-7856 (2015).

26. Chen, Y., Zhang, L., Wang, L., Guo, L. \& Liu, C. Living supramolecular polymerization of an AIE-active Ir(III) complex with irregular emission. Mater. Chem. Front. 5, 7808-7816 (2021).

27. Wang, X., et al. Cylindrical block copolymer micelles and co-micelles of controlled length and architecture. Science 317, 644-647 (2007).

28. Gadt, T., Ieong, N.S., Cambridge, G., Winnik, M.A. \& Manners, I. Complex and hierarchical micelle architectures from diblock copolymers using living, crystallization-driven polymerizations. Nat. Mater. 8, 144-150 (2009).

29. Gilroy, J.B., et al. Monodisperse cylindrical micelles by crystallization-driven living self-assembly. Nat. Chem. 2, 566-570 (2010).

30. Ganda, S. \& Stenzel, M.H. Concepts, fabrication methods and applications of living crystallization-driven self-assembly of block copolymers. Prog. Polym. Sci. 101, 101195 (2020).

31. Finnegan, J.R., et al. Stealth nanorods via the aqueous living crystallisation-driven self-assembly of poly(2-oxazoline)s. Chem. Sci. 12, 7350-7360 (2021).

32. Kim, H.J., Whang, D.R., Gierschner, J. \& Park, S.Y. Highly enhanced fluorescence of supramolecular polymers based on a cyanostilbene derivative and cucurbit[8]uril in aqueous solution. Angew. Chem. Int. Ed. Engl. 55, 15915-15919 (2016).

33. Kim, H.-J., Nandajan, P.C., Gierschner, J. \& Park, S.Y. Light-harvesting fluorescent supramolecular block copolymers based on cyanostilbene derivatives and cucurbit[8]urils in aqueous solution. Adv. Funct. Mater. 28, 1705141 (2017).

34. Yang, L., Bai, Y., Tan, X., Wang, Z. \& Zhang, X. Controllable supramolecular polymerization through host-guest interaction and photochemistry. ACS Macro Lett. 4, 611-615 (2015).

35. Yin, Z., et al. Dissipative supramolecular polymerization powered by light. CCS Chem. 1, 335-342 (2019).

36. Wu, H., et al. In situ photoconversion of multicolor luminescence and pure white light emission based on carbon dot-supported supramolecular assembly. J. Am. Chem. Soc. 141, 6583-6591 (2019).

37. Chen, X.M., Chen, Y., Yu, Q., Gu, B.H. \& Liu, Y. Supramolecular assemblies with near-infrared emission mediated in two stages by cucurbituril and amphiphilic calixarene for lysosome-targeted cell imaging. Angew. Chem. Int. Ed. Engl. 57, 12519-12523 (2018).

38. Chen, L.H., Huang, Z.H., Xu, J.F., Wang, Z.Q. \& Zhang, X. Controllable supramolecular polymerization through self-sorting of aliphatic and aromatic motifs. Polym. Chem. 7, 1397-1404 (2016).

39. Raeisi, M., et al. Sequence-specific self-assembly of positive and negative monomers with cucurbit[8] uril linkers. J. Am. Chem. Soc. 140, 3371-3377 (2018). 
40. Nandajan, P.C., Kim, H.J., Casado, S., Park, S.Y. \& Gierschner, J. Insight into water-soluble highly fluorescent low-dimensional host-guest supramolecular polymers: Structure and energy-transfer dynamics revealed by polarized fluorescence spectroscopy. J. Phys. Chem. Lett. 9, 3870-3877 (2018).

41. Correia, H.D., et al. Dynamic supramolecular polymers built from cucurbit[n]urils and viologens. Polym. Int. 68, 572-588 (2018).

42. Ni, X.L., Chen, S.Y., Yang, Y.P. \& Tao, Z. Facile cucurbit[8]uril-based supramolecular approach to fabricate tunable luminescent materials in aqueous solution. J. Am. Chem. Soc. 138, 6177-6183 (2016).

43. Hashim, P.K., Bergueiro, J., Meijer, E.W. \& Aida, T. Supramolecular polymerization: A conceptual expansion for innovative materials. Prog. Polym. Sci. 105, 101250 (2020).

44. Han, Y., Tian, Y., Li, Z. \& Wang, F. Donor-acceptor-type supramolecular polymers on the basis of preorganized molecular tweezers/guest complexation. Chem. Soc. Rev. 47, 5165-5176 (2018).

45. Yang, L., Tan, X., Wang, Z. \& Zhang, X. Supramolecular polymers: Historical development, preparation, characterization, and functions. Chem. Rev. 115, 71967239 (2015).

46. Fukui, T., et al. Direct observation and manipulation of supramolecular polymerization by high-speed atomic force microscopy. Angew. Chem. Int. Ed. Engl. 57, 15465-15470 (2018).

47. Shi, B., et al. Spontaneous formation of a cross-linked supramolecular polymer both in the solid state and in solution, driven by platinum(II) metallacycle-based host-guest interactions. J. Am. Chem. Soc. 141, 6494-6498 (2019).

48. Au-Yeung, H.-L., Leung, S.Y.-L. \& Yam, V.W.-W. Solvent-assisted supramolecular assembly of cyclotetrasiloxane-functionalized alkynylplatinum(II) terpyridine complexes. CCS Chem. 1, 464-475 (2019).

49. Liu, Y., Wang, Z. \& Zhang, X. Characterization of supramolecular polymers. Chem. Soc. Rev. 41, 5922-5932 (2012).

50. Rizvi, A., Mulvey, J.T., Carpenter, B.P., Talosig, R. \& Patterson, J.P. A close look at molecular self-assembly with the transmission electron microscope. Chem. Rev. 121, 14232-14280 (2021).

51. Liu, S., et al. Molecular motion in the solid state. ACS Macro Lett. 1, 425-431 (2019).

52. Barrio, J.D., et al. Emerging two-dimensional crystallization of cucurbit[8]uril complexes: From supramolecular polymers to nanofibers. J. Am. Chem. Soc. 141, 14021-14025 (2019).

53. Sun, M.J., et al. In situ visualization of assembly and photonic signal processing in a triplet light-harvesting nanosystem. J. Am. Chem. Soc. 140, 4269-4278 (2018).

54. Onogi, S., et al. In situ real-time imaging of self-sorted supramolecular nanofibres. Nat. Chem. 8, 743-752 (2016).

55. Yang, S. \& Choi, T.L. Rapid formation and real-time observation of micron-sized conjugated nanofibers with tunable lengths and widths in 20 minutes by living crystallization-driven self-assembly. Chem. Sci. 11, 8416-8424 (2020). 
56. Sarkar, A., et al. Self-sorted, random, and block supramolecular copolymers via sequence controlled, multicomponent self-assembly. J. Am. Chem. Soc. 142, 76067617 (2020).

57. Liu, S., et al. Manipulating solid-state intramolecular motion toward controlled fluorescence patterns. ACS Nano 14, 2090-2098 (2020).

58. Huang, C.B., et al. Real-time monitoring the dynamics of coordination-driven self-assembly by fluorescence-resonance energy transfer. J. Am. Chem. Soc. 139, 9459-9462 (2017).

59. Saigusa, H. \& Lim, E.C. Excimer formation in van der waals dimers and clusters of aromatic molecules. Acc. Chem. Res. 29, 171-178 (1996).

60. Pensack, R.D., Ashmore, R.J., Paoletta, A.L. \& Scholes, G.D. The nature of excimer formation in crystalline pyrene nanoparticles. J. Phys. Chem. C 122, 21004-21017 (2018).

61. Förster, T. \& Kasper, K. Ein konzentrationsumschlag der fluoreszenz. Z. Phys. Chem. 1, 275-277 (1954).

62. Yan, X.Z., Cook, T.R., Wang, P., Huang, F.H. \& Stang, P.J. Highly emissive platinum(II) metallacages. Nat. Chem. 7, 342-348 (2015).

63. Lou, X.Y. \& Yang, Y.W. Aggregation-induced emission systems involving supramolecular assembly. Aggregate 1, 19-30 (2020).

64. Mei, J., Leung, N.L.C., Kwok, R.T.K., Lam, J.W.Y. \& Tang, B.Z. Aggregationinduced emission: Together we shine, united we soar! Chem. Rev. 115, 1171811940 (2015).

65. Peng, Q. \& Shuai, Z. Molecular mechanism of aggregation-induced emission. Aggregate 2, e91 (2021).

66. Tu, Y., Zhao, Z., Lam, J.W.Y. \& Tang, B.Z. Aggregate science: Much to explore in the meso world. Matter 4, 338-349 (2021).

67. Lou, X.-Y. \& Yang, Y.-W. Manipulating aggregation-induced emission with supramolecular macrocycles. Adv. Optical Mater. 6, 1800668 (2018).

68. Chen, C., et al. Calixarene-based supramolecular AIE dots with highly inhibited nonradiative decay and intersystem crossing for ultrasensitive fluorescence imageguided cancer surgery. Angew. Chem. Int. Ed. Engl. 59, 10008-10012 (2020).

69. Yan, X., et al. Endo- and exo-functionalized tetraphenylethylene M12L24 nanospheres: Fluorescence emission inside a confined space. J. Am. Chem. Soc. 141, 9673-9679 (2019).

70. Ji, X., et al. A functioning macroscopic "rubik's cube" assembled via controllable dynamic covalent interactions. Adv. Mater. 31, e1902365 (2019).

71. Xu, S., Duan, Y. \& Liu, B. Precise molecular design for high-performance luminogens with aggregation-induced emission. Adv. Mater. 32, e1903530 (2020).

72. Feng, H.-T., Lam, J.W.Y. \& Tang, B.Z. Self-assembly of AIEgens. Coordin. Chem. Rev. 406, 213142 (2020).

73. Luo, J.D., et al. Aggregation-induced emission of 1-methyl-1,2,3,4,5pentaphenylsilole. Chem. Commun., 1740-1741 (2001).

74. Ma, S., et al. Organic molecular aggregates: From aggregation structure to emission property. Aggregate 2, e96 (2021). 
75. Shi, X., et al. Supramolecular polymerization with dynamic self-sorting sequence control. Macromolecules 52, 8814-8825 (2019).

76. Zhang, F., et al. Iodine-assisted assembly of helical coordination polymers of cucurbituril and asymmetric copper(II) complexes. Angew. Chem. Int. Ed. Engl. 44, 3402-3407 (2005).

77. Barrow, S.J., Kasera, S., Rowland, M.J., del Barrio, J. \& Scherman, O.A. Cucurbituril-based molecular recognition. Chem. Rev. 115, 12320-12406 (2015).

78. Wu, G., Olesińska, M., Wu, Y., Matak-Vinkovic, D. \& Scherman, O.A. Mining 2:2 complexes from 1:1 stoichiometry: Formation of cucurbit[8]uril-diarylviologen quaternary complexes favored by electron-donating substituents. J. Am. Chem. Soc. 139, 3202-3208 (2017).

79. Tang, X., et al. Supramolecularly catalyzed polymerization: From consecutive dimerization to polymerization. Angew. Chem. Int. Ed. Engl. 57, 8545-8549 (2018).

80. Liu, S.M., et al. The cucurbit[n]uril family: Prime components for self-sorting systems. J. Am. Chem. Soc. 127, 15959-15967 (2005).

81. Wu, H., et al. Ring-in-ring(s) complexes exhibiting tunable multicolor photoluminescence. J. Am. Chem. Soc. 142, 16849-16860 (2020).

82. Combes, S., et al. Triangular regulation of cucurbit[8]uril 1:1 complexes. J. Am. Chem. Soc. 141, 5897-5907 (2019).

83. Ni, X.L., et al. Cucurbit[n]uril-based coordination chemistry: From simple coordination complexes to novel poly-dimensional coordination polymers. Chem. Soc. Rev. 42, 9480-9508 (2013).

84. Hwang, W., et al. Hierarchical self-assembly of poly-pseudorotaxanes into artificial microtubules. Angew. Chem. Int. Ed. Engl. 59, 3460-3464 (2020).

85. Wei, P., et al. Molecular transmission: Visible and rate-controllable photoreactivity and synergy of aggregation-induced emission and host-guest assembly. Chem. Mater. 31, 1092-1100 (2019).

86. Wang, Q., et al. Color-tunable single-fluorophore supramolecular system with assembly-encoded emission. Nat. Commun. 11, 158 (2020).

87. Hua, B., et al. Pillar[5]arene-based solid-state supramolecular polymers with suppressed aggregation-caused quenching effects and two-photon excited emission. J. Am. Chem. Soc. 142, 16557-16561 (2020).

88. Zhang, Q.W., et al. Multicolor photoluminescence including white-light emission by a single host-guest complex. J. Am. Chem. Soc. 138, 13541-13550 (2016).

89. Biedermann, F., Vendruscolo, M., Scherman, O.A., De Simone, A. \& Nau, W.M. Cucurbit[8]uril and blue-box: High-energy water release overwhelms electrostatic interactions. J. Am. Chem. Soc. 135, 14879-14888 (2013).

90. Yao, Y., et al. Heteroepitaxial growth of multiblock ln-MOF microrods for photonic barcodes. Angew. Chem. Int. Ed. Engl. 58, 13803-13807 (2019).

91. Hudson, Z.M., Lunn, D.J., Winnik, M.A. \& Manners, I. Colour-tunable fluorescent multiblock micelles. Nat. Commun. 5, 3372 (2014).

92. Feng He, Torben Gädt, Ian Manners \& Winnik, M.A. Fluorescent "barcode" multiblock co-micelles via the living self-assembly of di- and triblock copolymers with a crystalline core-forming metalloblock. J. Am. Chem. Soc. 133, 9095-9103 
(2011).

93. Yang, X., Wang, R., Kermagoret, A. \& Bardelang, D. Oligomeric cucurbituril complexes: From peculiar assemblies to emerging applications. Angew. Chem. Int. Ed. Engl. 59, 21280-21292 (2020). 